\title{
Euromaidán Y La Crisis Política De UCRANia: ANTECEDENTES Y PERSPECTIVAS
}

\section{Hanna Kulyk ${ }^{1}$}

Resumen: Euromaidán es el nombre dado a una serie de manifestaciones que comenzó a finales del año 2013 en Ucrania, cuando el ex presidente Victor Yanukovych declaró la suspensión de la firma del Acuerdo de Asociación con la Unión Europea. Como resultado, miles de personas salieron a las calles de Kiev para mostrar su desacuerdo con la decisión del Gobierno. De esta manera, el Euromaidán, que empezó siendo un movimiento social, acabó provocando una confrontación entre los ciudadanos y el Gobierno ucraniano, situación que derivó en un conflicto militar con Rusia en el este de Ucrania. Esta situación marcó el comienzo de la actual crisis política del país. En este artículo se presentará el contexto histórico amplio como también la cronología actual de la crisis ucraniana, desde noviembre de 2013 hasta mayo de 2019. Todos los acontecimientos de esta crisis ucraniana deben considerarse como un único proceso tal y como se muestra en la siguiente secuencia: Euromaidán, anexión de Crimea, fase activa del conflicto militar en Dombás (hasta la firma del Segundo Protocolo de Minsk en 2015), fase pasiva del conflicto militar en Dombás y militarización del Mar Negro. Finalmente, se presentarán las conclusiones y las perspectivas para el futuro.

Palabras clase: Euromaidán; crisis política; relaciones Ucrania-Rusia; guerra en Dombás

${ }^{1}$ Máster en Sociología. Universitat de Barcelona; hanna.kulyk@gmail.com 
Abstract: Euromaidan is the name given to a series of demonstrations that began at the end of 2013 in Ukraine, when former President Victor Yanukovych declared the suspension of the signing of the Agreement about the Association with the European Union. As a result, thousands of people started to protest on the streets of Kiev to show their disagreement with the decision of the government. Thus, Euromaidan, which began as a social movement, ended up causing a confrontation between citizens and the Ukrainian government, which led to the military conflict with Russia in Eastern Ukraine. This situation marked the beginning of the current political crisis in the country. The article presents the broad historical context of the conflict, as well as the current chronology of the Ukrainian crisis, from November 2013 to May 2019. All the events of this Ukrainian crisis should be considered as a single process, as shown in the following sequence: Euromaidan, annexation of Crimea, active phase of military conflict in Donbass (until the signing of the Second Minsk Protocol in 2015), passive phase of the military conflict in Donbass, and militarization of the Black Sea. Finally, the conclusions and future prospects will be presented.

Keywords: Euromaidan; political crisis; Ukraine-Russia relations; war in Donbass.

Resumen: Euromaidán és el nom que rep una sèrie de manifestacions que va començar a final de l'any 2013 a Ucraïna, quan l'expresident Victor Yanukovych va declarar la suspensió de la signatura de l'Acord d'Associació amb la Unió Europea. Com a conseqüència, milers de persones van sortir als carrers de Kiev per mostrar el seu desacord amb la decisió del Govern. D'aquesta manera, l'Euromaidan, que va començar sent un moviment social, va acabar per provocar una confrontació entre els ciutadans i el Govern ucraïnès, situació que va 
derivar en un conflicte militar amb Rússia a l'est d'Ucraïna. Aquesta situació va marcar el principi de l'actual crisi política del país. En aquest article es presentarà el context històric ampli, a més de la cronologia actual de la crisi ucraïnesa, des de novembre de 2013 fins a maig de 2019. Tots els esdeveniments han de considerar-se com un únic procés, tal com mostra la següent seqüència: Euromaidan, annexió de Crimea, fase activa del conflicte militar al Dombass (fins a la signatura del Segon Protocol de Minsk el 2015), fase passiva del conflicte militar al Dombass i militarització del Mar Negre. Finalment, s'hi presentaran les conclusions i les perspectives per al futur.

Palabras clave: Euromaidan; crisi política; relacions Ucraïna-Russia; guerra al Dombass

Introducción e historia del fenómeno

Ucrania es un país situado al este de Europa, con una nación históricamente dividida en dos partes: la noroeste, donde la lengua principal es el ucraniano, y la sudoeste, con el ruso como idioma dominante. Esta división ha sido establecida debido a las complejas relaciones económicas y sociales que ha mantenido Ucrania a lo largo de su historia. Por un lado, gran parte de Ucrania estuvo bajo control ruso a lo largo de los últimos 400 años, primero, formando parte del Imperio Ruso y, después, de la Unión Soviética. Por otro lado, las regiones de Ucrania occidental, durante diferentes períodos, estuvieron bajo el control del Imperio Austríaco, el Reino Húngaro y Polonia.

Cabe señalar que el gobierno autoritario del Imperio Ruso y el gobierno totalitario de la Ucrania soviética fueron menos democráticos que los gobiernos austrohúngaro y polaco (Katchanovski, 2016). La política de los gobiernos del Imperio Ruso y, posteriormente, de la Unión Soviética, se caracterizaron por las campañas antinacionalistas. Estas campañas iban en contra de los historiadores y líderes políticos ucranianos, de las formaciones militares ucranianas que luchaban en favor de la independencia, así como de la Iglesia griego-católica 
ucraniana, en definitiva, en contra de todo aquel que cuestionase la jerarquía establecida por los rusos, en la que ellos eran los "hermanos mayores" y los ucranianos eran los malorossy ("pequeños rusos"). Aquellos que no eran leales a esta jerarquía establecida en las épocas zaristas y soviéticas fueron definidos como "agentes de Austria", como "nacionalistas burgueses" y como "fascistas" (Kuzio, 2016). Esta misma categorización se observa en los discursos de la Rusia actual, en los que Vladimir Putin se refiere a los rusos como a un pueblo multinacional, en el que las personas se caracterizan, no tanto por la nacionalidad, etnicidad o lengua, sino por un principio moral superior, que les hace pertenecer al mundo ruso (russkiy mir). Un claro ejemplo del cariz de estos discursos es el uso del término Novorossia (Nueva Rusia), empleado por Putin, para referirse al centro, este y sudeste de Ucrania, ya que, según él, estas regiones, "por supuesto, están arraigadas al estado ruso y tienen una mentalidad diferente" en comparación con las regiones del oeste de Ucrania, donde, debido a su compleja historia, se observa un "renacimiento de neonazismo" (Putin, 2014b).

Los objetivos del gobierno soviético con respecto a Ucrania estaban orientados a la eliminación de la identidad nacional, que consistía en la reeducación de los pueblos de la URSS para identificarlos con el presente soviético y el pasado imperial ruso, la rusificación generalizada y la imposición de símbolos e ideas comunistas, así como también la prohibición de la lengua ucraniana y de la Iglesia griego-católica, muchos de cuyos seguidores vivían en el oeste de Ucrania. En los años 1932-1933 los soviéticos cometieron un crimen aún más grave contra el pueblo ucraniano -el Holodomor, una hambruna masiva que llevó a la muerte de miles de personas-. Todos estos hechos históricos se han convertido en los conflictos no resueltos entre Ucrania y Rusia, que a menudo están utilizados en los discursos pro-europeos actuales. 
Euromaidán: ¿por qué la gente salió a la calle?

La lucha de los ciudadanos ucranianos por sus derechos, llamada "Euromaidán", o "Revolución de la dignidad" se ha convertido en el evento más importante de la historia moderna de Ucrania, que ha modificado la conciencia colectiva de los ucranianos (Kyrydon, 2015).

La causa principal del Euromaidán fue el desacuerdo con el gobierno de Yanukovych después del aplazamiento del acuerdo sobre la asociación y libre comercio con la Unión Europea (Katchanovski, 2016), pero entre otras razones también se puede destacar el abandono sistemático de las necesidades y problemas de los ciudadanos y de la sociedad ucraniana, así como la falta de respeto por parte de las autoridades (Kyrydon, 2015), además de la corrupción y los problemas sociales y económicos (Gaidai \& Liubaretc, 2016).

Varios autores consideran que el Euromaidán (llamado igualmente Revolución de la Dignidad) comparte muchos objetivos con la Revolución Naranja, que tuvo lugar en Ucrania en el año 2004. En 2004 Viktor Yanukovych y su Partido de las Regiones intentaron ganar las elecciones presidenciales falsificando los resultados a su favor (Katchanovski, 2016; Paniotto, 2013; Shveda \& Ho Park, 2016). Sin embargo, las elecciones presidenciales del 2010, donde Yanukovych ganó por poca diferencia, fueron justas y sin violaciones significativas de la ley. Después de llegar al poder, el presidente Yanukovych confirmó la política para la integración de Ucrania en la Unión Europea, sin embargo, los discursos de Yanukovych y los diputados de su Partido de las Regiones indicaban la orientación del presidente y del partido hacia Rusia (Paniotto, 2013). Según el análisis de Katchanovski (2016), el sistema político ucraniano durante el gobierno de Yanukovych en Ucrania la democracia decreció en favor de una autocracia. Entre los años 2010 y 2014 el presidente y las personas más 
cercanas a él aumentaron su riqueza; en particular, el hijo mayor de Yanukovych, que se convirtió en una de las personas más ricas de Ucrania (Kyrydon, 2015). Cuando en 2013 el presidente negó firmar el Acuerdo de Asociación de Ucrania con la UE, muchos ucranianos consideraron este hecho como un acto de rendición a la construcción de una democracia e instauración de un modelo político autocrático u oligárquico en el país. En la conciencia de los ucranianos, la Unión Europea estaba asociada con "el estado de derecho, respeto por la personalidad mediante reglas transparentes de funcionamiento del sistema político" (Paniotto, 2013). Luchar por estos principios democráticos fue la principal razón de los primeros manifestantes para salir a las calles de Kiev.

\section{Opinión pública sobre el Euromaidán}

Diversos estudios muestran una división en la opinión de los ucranianos sobre el Euromaidán. Según los resultados de la encuesta realizada entre el 9 y el 20 de noviembre del año 2013 por el Instituto Internacional de Sociología de $\mathrm{Kiev}^{2}$, el 54\% de la población ucraniana estaba a favor de unirse a la Unión Europea y un $46 \%$ en contra. En la pregunta cerrada, donde las personas podían elegir solo una opción entre Unión Europea y Unión Aduanera con Rusia, Bielorrusia y Kazajistán, la opinión estaba dividida casi por igual: 51\% vs. un 49\%. En otro estudio realizado por el mismo Instituto entre el 7 y el 8 de diciembre del $2013^{3}$, las principales razones por las que la gente salió a la calle fueron: la agresión por parte de las fuerzas de seguridad a los manifestantes durante la noche del 30 de noviembre $(70 \%$ de los encuestados), la negativa de Yanukovych a firmar el Acuerdo de

2La encuesta contaba con la siguiente muestra: 2011 personas mayores de 18 años de todas las regiones de Ucrania (incluyendo la cuidad de Kiev y Crimea). El error estadístico no supera el 3,3\%.

${ }^{3}$ En este estudio 1037 manifestantes fueron entrevistados mediante entrevistas personales. El error de muestreo teórico no supera el 3,2\%. 
Asociación con la Unión Europea (54\% de los encuestados) y el deseo de cambiar la vida en Ucrania (50\% de encuestados) (Paniotto, 2013).

Según el estudio realizado por el Instituto de Tecnologías Sociales "Sociopolis" en enero 20144, el 37,7\% de los encuestados creía que Ucrania debía unirse a la Unión Europea y el 28,9\% de los encuestados preferían la entrada a la Unión Aduanera. Sin embargo, un porcentaje significativo $(25,1 \%)$ creía que Ucrania no debía unirse ni a la Unión Europea ni a la Unión Aduanera. Según este estudio, los motivos principales de la gente para salir a la calle fueron la negativa de Yanukovych a firmar el Acuerdo de Asociación con la UE (51,4\% de los encuestados) y una situación sociopolítica y socioeconómica desfavorable en el país (41,7\% de los respondientes) (Sociopolis, 2014).

Aunque la opinión pública sobre el futuro geopolítico de Ucrania al igual que los motivos de los manifestantes, ha ido cambiando durante diferentes etapas de Euromaidán, sí pueden destacarse algunas características generales en las demandas de los manifestantes, como el deseo por la justicia y la protección de la dignidad de las personas. También cabe señalar, que la opinión pública no ha sido homogénea en relación con el futuro geopolítico de Ucrania. Una gran parte de la población, especialmente las personas de las regiones del este de Ucrania y Crimea apoyaban la adhesión del país a la Unión Aduanera. Esta división entre las opiniones empujó a la gente a participar en las manifestaciones activas y a veces violentas, Euromaidán y Antimaidán, que han marcado el comienzo de la crisis política en Ucrania. La crisis se ha convertido en uno de los desafíos más complejos, no solo para Ucrania, sino también para la comunidad mundial. Las protestas

${ }^{4} \mathrm{La}$ encuesta contaba con la siguiente muestra: 1650 personas mayores de 18 años de80asentamientos que representan a todas las regiones de Ucrania y todas las categorías de asentamientos según su tipo y población. El error estadístico no supera el 2,4\%. 
masivas, el cambio de régimen y la guerra han producido transiciones políticas que también han transformado la estructura social del país.

Para realizar este trabajo las principales fuentes seleccionadas, por su calidad y seguimiento de la noticia, han sido varios artículos científicos en ucraniano, ruso, inglés y español, los informes oficiales de las Naciones Unidas, las encuestas sobre la opinión pública y el diario Ukraiinska pravda.

La crisis ucraniana: cronología de los acontecimientos

A continuación, se presenta la cronología de los principales acontecimientos de la crisis ucraniana, desde noviembre del 2013 hasta mayo del 2019. Todos los eventos de la crisis ucraniana deben considerarse como un único proceso dentro de la siguiente secuencia: Euromaidán - Anexión de Crimea - fase activa del conflicto militar en Dombás (hasta la firma del Segundo Protocolo de Minsk en 2015) fase pasiva del conflicto militar en Dombás y militarización del Mar Negro.

\begin{tabular}{|ll|}
\hline Euromaidán & \multicolumn{4}{|c|}{2013} \\
\hline 21.11. & Inicio del Euromaidán. El presidente Viktor Yanukovych \\
& anuncia la suspensión del acuerdo de asociación con la Unión \\
& Europea en la cumbre de Vilna. Como protesta, 1.500 \\
& personas salen al Maidán Nezalézhnosti5 en Kiev, en su \\
& mayoría activistas públicos, periodistas, políticos de la \\
& oposición y estudiantes universitarios ("Maidan. El \\
& nacimiento," 2014).
\end{tabular}

\footnotetext{
${ }^{5}$ Maidán Nezaléz̧hnosti (Plaza de la Independencia) es la plaza central de Kiev, donde ocurrieron las principales manifestaciones durante el Euromaidán. La palabra "Euromaidán” hace referencia al nombre de esta plaza.
} 


\begin{tabular}{|l}
\hline manifestaciones en muchas ciudades ucranianas y en otras \\
ciudades del mundo, como Paris, Ginebra, Múnich, Praga, \\
Toronto). El 24 de noviembre en Kiev, entre 50 y 100 mil \\
personas se reunieron en el centro de la ciudad (Paniotto, \\
2013), teniendo lugar los primeros enfrentamientos entre los \\
manifestantes y la policía y los titushki (grupos de jóvenes que \\
apoyan al gobierno y hacen provocaciones en el Maidán. \\
\hline 26.11.-29.11. \\
Participación de los estudiantes en las manifestaciones pro- \\
europeas, cuya demanda principal es la firma del Acuerdo de \\
Asociación con la UE. \\
Se celebra en Vilna la Cumbre de la "Asociación Oriental", en \\
la que la delegación ucraniana no firma el Acuerdo de \\
Asociación con la UE y la entrada en la zona de libre \\
comercio. Los manifestantes de Euromaidán exigen la \\
renuncia inmediata de Yanukovych. \\
A las cuatro de la mañana en el Maidán más de doscientos \\
combatientes de las fuerzas especiales Berkut disuelven el mitin \\
de los partidarios de la integración europea. Como resultado, \\
muchas personas resultan heridas, de las 7 son hospitalizadas \\
(Paniotto, 2013). \\
Al mediodía empieza la protesta organizada por los políticos \\
de la oposición. El número de participantes es de unas 500 mil \\
personas. Después del mitin y de una caminata masiva, los \\
manifestantes logran apoderarse del edificio del Ayuntamiento \\
de Kiev y ocupan el edificio de la Casa de los Sindicatos, \\
donde ubican la sede del Euromaidán. Las fuerzas políticas de \\
derecha, también afines a la entrada en la Unión Europea, \\
intensifican sus acciones, en particular cometen un acto de \\
vandalismo contra el monumento de Lenin en Kiev (Gaidai \& \\
Liubaretc, 2016; Kyrydon, 2015; Paniotto, 2013). \\
Continúan las protestas. Las principales demandas de los
\end{tabular}


manifestantes son la renuncia del gobierno, la celebración de elecciones presidenciales y gubernamentales extraordinarias, la liberación de los prisioneros que han sido encarcelados después de las manifestaciones del 1 de diciembre.

11.12. A la una de la madrugada las fuerzas especiales, junto a las tropas internas lanzan una acción masiva para expulsar a los manifestantes de sus posiciones. Logran eliminar algunas de las barricadas, pero no pueden dispersar completamente a los manifestantes ("La policía puede volver a utilizar la fuerza contra manifestantes - Ministerio del Interior,” 2013)

\section{4}

16.01. Verkhovna Rada de Ucrania adopta un paquete de leyes, que prohíbe las manifestaciones y asambleas, aumenta la responsabilidad por determinados delitos y fortalece la protección de los jueces y representantes de los cuerpos de seguridad (Kyrydon, 2015). Estas leyes han sido conocidas como leyes dictatoriales y pueden considerarse como una acción del gobierno de mantener el poder y convertir el sistema político en una autocracia (Shveda \& Ho Park, 2016).

19.01.-21.01. Se fortalece el ánimo radical de los manifestantes, se produce un gran número de enfrentamientos entre manifestantes y fuerzas de seguridad. La demanda principal de los manifestantes es derogar las leyes del 16 de enero.

22.01. "El día de la reunión sangrienta". Alrededor de las ocho de la mañana, los combatientes de las fuerzas especiales Berkut atacan a los manifestantes en la calle Hrushevsky ("Berkut empezó el ataque en Hrushevsky," 2014). Como resultado, tres participantes de Euromaidán han sido asesinados (Shveda \& Ho Park, 2016).

22.01.-18.02. Se mantienen varias reuniones pacíficas entre los representantes de la oposición y Yanukovych, con la 
intervención de políticos europeos del gobierno de Polonia, de Francia, de Alemania y el presidente de la Federación Rusa. Los políticos acuerdan realizar elecciones presidenciales en diciembre del 2014. Sin embargo, los resultados de estas reuniones no satisfacen a las los manifestantes, que insisten en establecer la fecha el 21 de febrero del 2014 (Shveda \& Ho Park, 2016).

18.02.-20.02 A partir del 18 de febrero las fuerzas del gobierno de Yanukovych empiezan a usar armas de fuego contra los manifestantes ("Titushky dispararon a dos manifestantes testigos," 2014). Durante tres días, 88 participantes del Euromaidán han sido asesinados. Si se cuentan las muertes posteriores, incluyendo a aquellos que murieron a causa de las lesiones sufridas durante las protestas, se obtiene un total de 113 muertes, a las que se hace referencia como los "Cien celestiales" (Shveda \& Ho Park, 2016).

21.02. Los políticos de la oposición y el presidente Yanukovych firman el acuerdo "Sobre la regulación de la crisis política en Ucrania", que provee la reelección del presidente antes de diciembre del 2014. Sin embargo, los participantes del Euromaidán rechazan dicho acuerdo y asaltan el Palacio Presidencial, exigiendo la renuncia inmediata del presidente. Yanukovych huye de Kiev (Kyrydon, 2015; Shveda \& Ho Park, 2016).

22.02. El parlamento de Ucrania reconoce que Viktor Yanukovych se retira del ejercicio de autoridad constitucional, entonces es apartado de su cargo como presidente de Ucrania (Kyrydon, 2015).

Ucrania declara el retorno a la Constitución de 2004, que convierte a Ucrania en una república parlamentaria (Kudryashev, 2015; Kyrydon, 2015). El nuevo Gobierno de 
Transición está reconocido por los actores internacionales. Esta fecha puede ser considerada como el fin del Euromaidán (Kyrydon, 2015; Shveda \& Ho Park, 2016).

Anexión de Crimea. El cambio de gobierno provocó en Crimea manifestaciones de los dos bandos opositores: los que apoyan la integración en la UE y los que apoyan la integración en Rusia.

27.02. El parlamento de Crimea convoca un referéndum para el 25 de mayo. La pregunta de este referéndum es: “¿La República Autónoma de Crimea tiene autonomía estatal y es parte de Ucrania sobre la base de tratados y acuerdos?" (sí o no).

El nuevo gobierno pro-ruso toma el poder. ("El Parlamento de Crimea nombró a un referéndum y dispersa el gobierno," 2014).

01.03. El nuevo gobierno de Crimea cambia la fecha del referéndum para el 30 de marzo.

06.03. El nuevo gobierno de Crimea decide hacer el referéndum el 16 de marzo con 2 preguntas. La primera, caboga usted por la entrada de Crimea a la Federación Rusa como una federación?, y la segunda, ¿aboga usted por la restauración de la Constitución de Crimea de 1992? (según esta Constitución, Crimea es una república autónoma de Ucrania) ("El consejo de Crimea ha nombrado un referéndum para unirse a la Federación Rusa para el 16 de marzo.," 2014)

16.03. Se lleva a cabo el referéndum sobre el estado de Crimea. Según los datos oficiales proporcionados por los medios de comunicación rusos, el 96,77\% de la población de Crimea votó por la reunificación de los respectivos territorios con la Federación Rusa. Sin embargo, el referéndum en Crimea no ha sido reconocido ni por el gobierno ucraniano, ni por ningún país de la comunidad internacional (Kyrydon, 2015).

18.03. El presidente Vladímir Putin firma el acuerdo sobre la 
aceptación de Crimea en la Federación Rusia.

Conflicto militar en el Este de Ucrania 2014-2015 (fase activa). Después de la anexión de Crimea, los grupos pro-rusos de regiones del este de Ucrania comenzaron a manifestarse en la calle, lo que provocó hostilidades en la región.

06.04. Los grupos nacionalistas rusos ultra-reaccionarios comienzan a llevar a cabo acciones activas, en particular ocupan varios edificios administrativos en Donetks (Nagornyak, 2015).

07.04. El presidente interino de Ucrania, Oleksandr Turchínov, firma una declaración sobre la "Operación Antiterrorista" (ATO) en el este de Ucrania (Mityukov, 2016).

12.04. Los grupos separatistas de Donetsk ocupan las ciudades de Slavyansk, Kramatorsk y Artemivsk.

15.04. Las tropas gubernamentales comienzan a asaltar la ciudad de Slavyansk. Este día puede ser considerado como el inicio de la guerra entre los militares ucranianos y los separatistas prorusos.

02.05. En Odessa (sur de Ucrania) durante un partido de fútbol, ocurren las confrontaciones entre las fuerzas pro-ucranianas y pro-rusas. Como resultado 42 personas mueren y más de 200 resultan heridas ("Durante los disturbios en Odessa, 42 personas murieron. El jefe de policia fue despedido,” 2014).

11.05. Referéndum ilegal sobre la independencia de la República Popular de Donetsk y la República Popular de Luhansk.

26.05.- $\quad$ Combate por el aeropuerto de Donetsk (200 muertos). 21.01.2015

05.06.-29.08. Combate por Savur Mogyla (49 muertos).

14.06. Los separatistas pro-rusos derriban el avión Il-76 del ejército ucraniano en el aeropuerto de Luhansk (49 muertos).

17.07. Catástrofe del "Boeing 777" de Malasia Airlines en la región de Donetsk (298 muertos). 


\begin{tabular}{|c|c|}
\hline $\begin{array}{l}\text { 28.07.- } \\
18.02 .2015\end{array}$ & Combate por Debaltseve (179 muertos). \\
\hline 05.09 . & Firma del Protocolo de Minsk para acabar la guerra. \\
\hline & 2015 \\
\hline 13.01.2015 & $\begin{array}{l}\text { Bombardeo de un autobús con pasajeros civiles en un puesto } \\
\text { de control cerca de la ciudad de Volnovaha ( } 12 \text { muertos). }\end{array}$ \\
\hline 24.01. & Bombardeo de la ciudad de Mariupol (30 muertos). \\
\hline 12.02 . & $\begin{array}{l}\text { Firma del Segundo Protocolo de Minsk para acabar la guerra } \\
\text { ("Tres años de guerra en Donbas: eventos clave de ATO," } \\
\text { 2017) }\end{array}$ \\
\hline 03.03 . & $\begin{array}{l}\text { La oficina de derechos humanos de la ONU publica un } \\
\text { Informe sobre la situación de los derechos humanos en } \\
\text { Ucrania. Según este informe, desde el inicio de la guerra en } \\
\text { abril } 2014 \text { hasta el } 15 \text { de febrero del } 2015 \text {, al menos } 5.665 \\
\text { personas murieron y } 13.961 \text { resultaron heridas en el este de } \\
\text { Ucrania - } 19.626 \text { personas en total. Estas cifras están basabas } \\
\text { en los números de los damnificados reportados (Report on the } \\
\text { buman rights situation in Ukraine from } 1 \text { December } 2014 \text { to } 15 \\
\text { February 2015, 2015) }\end{array}$ \\
\hline \multicolumn{2}{|c|}{$\begin{array}{l}\text { Conflicto militar en el este de Ucrania (fase pasiva), militarización del } \\
\text { Mar Negro. Desde } 2015 \text { hasta ahora las batallas continúan en todos los } \\
\text { frentes, pero con menos intensidad. Los acuerdos de Minsk de los años } \\
2014 \text { y 2015, han contenido, pero no han detenido los combates (Sasse \& } \\
\text { Lackner, 2018). Casi todos los días hay datos sobre muertos y heridos } \\
\text { entre militares y personas civiles en el este de Ucrania. }\end{array}$} \\
\hline 15.11. & $\begin{array}{l}\text { Hasta el } 15 \text { de noviembre de } 2015 \text { el número estimado de } \\
\text { damnificados por el conflicto en el este de Ucrania es de } \\
29.830 \text { personas ( } 9.098 \text { muertos y } 20.732 \text { heridos) (Report on the } \\
\text { buman rights situation in Ukraine from } 16 \text { August to } 15 \text { November } \\
\text { 2015, 2015). }\end{array}$ \\
\hline & 2010 \\
\hline
\end{tabular}




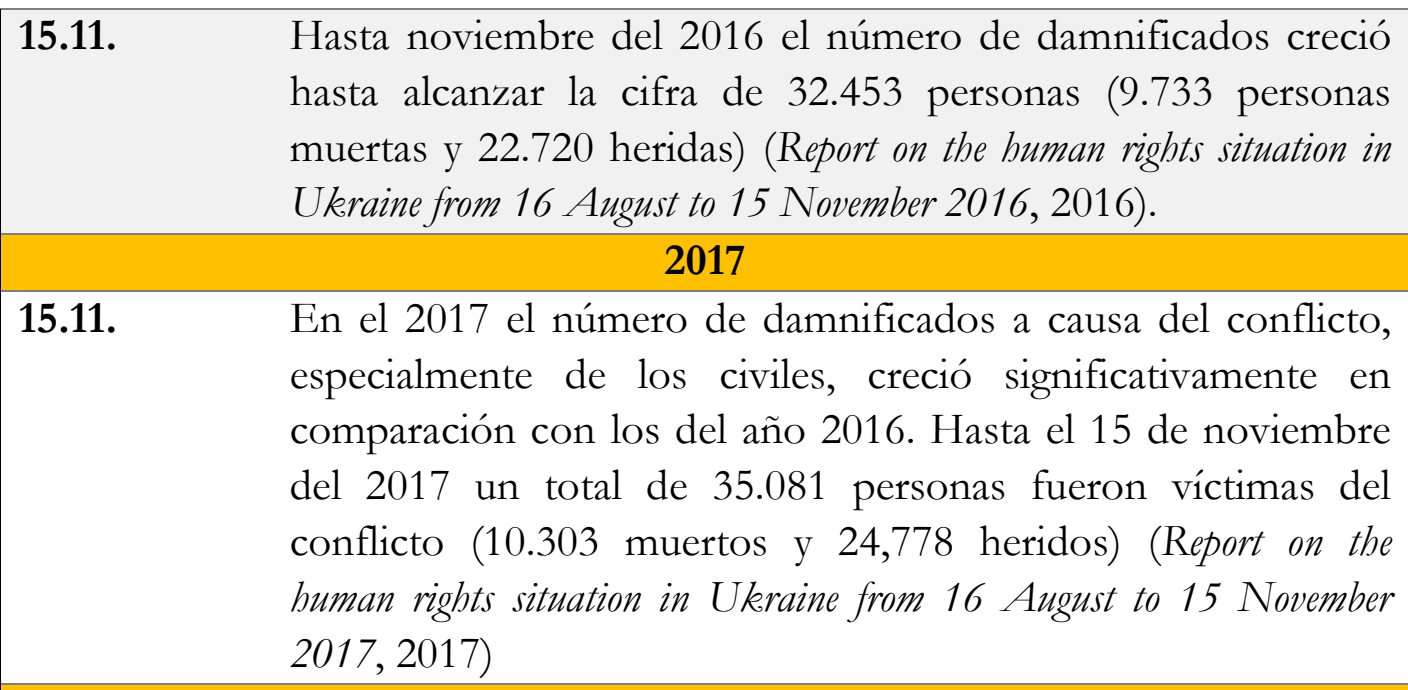

\section{8}

15.11. En 2018 el número de civiles damnificados durante el conflicto en el este de Ucrania disminuye en comparación con el 2017. Sin embargo, según el informe de la Oficina de los Derechos Humanos de la ONU, el conflicto sigue sin ningún final previsto. Las violaciones graves de derechos humanos, como la tortura y la detención arbitraria, continúan sin impunidad (Report on the human rights situation in Ukraine from 16 August to 15 November 2018, 2018).

25.11. Durante todo el año 2018 la tensión en la región del Mar Negro fue en aumentar. El 25 de noviembre cerca del Estrecho de Kerch en el Mar Negro, barcos fronterizos del Servicio de Seguridad ruso abrieron fuego contra un grupo naval de la Armada de Ucrania. Los militares rusos hieren a seis militares ucranianos y se apoderan de 3 barcos ucranianos ("La guerra en el estrecho de Kerch: barcos, aviones y captura de barcos ucranianos," 2018).

26.11. En diez regiones de Ucrania se introduce la ley marcial por primera vez durante todo el periodo del conflicto. La razón 


\begin{tabular}{|c|c|}
\hline & $\begin{array}{l}\text { principal - el acto militar de Rusia contra los barcos } \\
\text { ucranianos en el Estrecho de Kerch. Por la primera vez se } \\
\text { habla sobre un conflicto directo entre Ucrania y Rusia (“30 } \\
\text { días de la guerra. ¿Cuál fue el motivo de la introducción de la } \\
\text { ley marcial en Ucrania?,” 2018). }\end{array}$ \\
\hline 26.12 . & Termina la ley marcial en Ucrania. \\
\hline & 2019 \\
\hline 06.01 . & $\begin{array}{l}\text { Un barco de desembarco estadounidense entra por la primera } \\
\text { vez en el Mar Negro para "realizar operaciones para garantizar } \\
\text { la seguridad y la estabilidad en Europa" ("El barco de } \\
\text { desembarco estadounidense USS FortMcHenry entró en el } \\
\text { Mar Negro," 2019). }\end{array}$ \\
\hline 09.01. & $\begin{array}{l}\text { Por primera vez desde la ocupación de Crimea un barco, } \\
\text { perteneciente a la armada rusa entra al Mar Negro ("Una } \\
\text { marina naval de la Flota del Norte de la Federación Rusa entró } \\
\text { en el Mar Negro," 2019). }\end{array}$ \\
\hline 20.02 . & $\begin{array}{l}\text { Sigue la militarización del Mar Negro. Un destructor } \\
\text { estadounidense entra en el Mar Negro para realizar } \\
\text { entrenamientos internacionales; la armada rusa está } \\
\text { continuamente vigilando al destructor estadounidense ("Un } \\
\text { destructor estadounidense entró en el mar negro," 2019). }\end{array}$ \\
\hline 01.03.-20.04. & $\begin{array}{l}\text { Tanto los barcos de la OTAN como los militares rusos } \\
\text { participan en entrenamientos militares en el Mar Negro. }\end{array}$ \\
\hline 24.04 . & $\begin{array}{l}\text { Vladímir Putin firma un decreto sobre un procedimiento } \\
\text { simplificado para dar la ciudadanía rusa a los residentes de los } \\
\text { distritos ocupados en las regiones de Donetsk y Luhansk. Este } \\
\text { hecho va en contra de los Acuerdos de Minsk y amenaza a la } \\
\text { soberanía de Ucrania ("Putin firmó un decreto sobre la } \\
\text { emisión de pasaportes rusos a los residentes de regiones de } \\
\text { Donetsk y Luhansk," 2019). }\end{array}$ \\
\hline
\end{tabular}




\section{Conclusiones}

Al revisar la cronología de la crisis política ucraniana, cabe señalar que durante los 5 años de su duración han tenido lugar varias transformaciones en los dos lados del conflicto. El Euromaidán, que comenzó como un movimiento social, provocó un enfrentamiento entre los ciudadanos y el gobierno ucraniano y derivó en lo que se convirtió en un conflicto militar en el este de Ucrania, tensando las relaciones con Rusia.

Las consecuencias para los civiles de la guerra en Dombás son terribles. Según los datos aproximados de la Oficina de los Derechos Humanos de la ONU, desde el inicio de la guerra en abril del 2014 hasta febrero del 2019, al menos 3.321 civiles murieron y unas 7.000 personas fueron heridas por motivos relacionados con el conflicto. Más de 5 millones de personas continúan sufriendo las consecuencias graves del conflicto, esto incluye 1,3 millones de desplazados internos registrados y personas que viven en los pueblos en la línea de conflicto en el este de Ucrania. Una docena de civiles murieron en las primeras semanas del 2019, principalmente debido a graves complicaciones de salud mientras cruzaban la línea de conflicto. Más de 40,000 viviendas civiles en las regiones de Donetsk y Luhansk han sido dañadas o destruidas como resultado de las hostilidades en ambos lados de la línea del frente. Otros desafíos para los civiles son la falta de calefacción adecuada durante los meses de invierno, el impago de pensiones y la restricción de la libertad de expresión y de los medios de comunicación (Report on the human rights situation in Ukraine 16 November 2018 to 15 February 2019, 2019).

¿Cuál es la situación actual? A principios del año 2019, no había duda de que la situación se podía caracterizar como de conflicto militar 
entre Rusia y Ucrania. Varios autores (Radkovets Y. I., 2014; Tipaldou \& Casula, 2018) han utilizado las palabras "guerra" o "guerra híbrida" para describir la anexión de Crimea, la aparición de los militares con pasaportes rusos entre los separatistas de Donetsk y la militarización activa del Mar Negro. Según Radkovets, en los conflictos armados, donde se usan tecnologías de guerra híbrida, el lado defensor está luchando contra varios tipos de formaciones extremistas y terroristas en su territorio, las cuales son organizadas, provistas y guiadas desde otros países. Por diferentes razones (principalmente seguridad internacional y económica) el país defensor no puede implementar las formas activas de respuesta al agresor. Como resultado, hablamos de un conflicto, en el que se produce una destrucción gradual de la estructura económica y social de la sociedad (Radkovets Y. I., 2014).

En mayo del 2019 la situación en el este de Ucrania, al igual que en el Mar Negro continúa siendo inestable, lo que podría provocar una nueva escalada del conflicto. Según un estudio de Tipaldou y Casula (2018), "el discurso político oficial ruso ha incorporado rasgos de marcado tinte populista en el contexto de la crisis de Crimea y posterior guerra en Dombás". Para Putin los ucranianos y los rusos pertenecen al mismo pueblo que comparte los símbolos del pasado y enemigos comunes. En estos discursos Putin se representa como un padre-salvador de todas las naciones del "mundo ruso". En el 2014, comentando la anexión "pacifica" de Crimea, el presidente de la Federación Rusa explicó que "no tenía otra opción que respetar la voluntad de los habitantes de Crimea” (Putin, 2014a). En el año 2019 la política de simplificación de procedimiento para obtener los pasaportes rusos para los residentes del este de Ucrania puso un discurso parecido sobre la mesa. En particular, el asistente del presidente de Rusia, Vladislav Surkov, declaró que este decreto es "un deber de Rusia a las personas que hablan y piensan en ruso" ("Surkov sobre los pasaportes de la Federación Rusa en regiones de Donetsk y 
Luhansk: es un deber a los hablantes de ruso," 2019). Los discursos oficiales rusos tienen características pronunciadas de populismo, donde Putin aparece representado como el líder de Rusia y Ucrania, de la integración euroasiática y de recreación de la Unión Soviética (Tipaldou \& Casula, 2018). Estos discursos podrían llevar a más conflictos entre Ucrania y Rusia.

La crisis ucraniana es una crisis de democracia, producida por una lucha entre las antiguas y las nuevas formas de vida. Desde el comienzo del Euromaidán sus participantes se declararon en contra del régimen autoritario de Yanukovych y trataron de romper con los símbolos del totalitarismo soviético, por ejemplo, el desmantelamiento de monumentos de Lenin y otros monumentos soviéticos (Gaidai \& Liubaretc, 2016). Sin embargo, la evidencia muestra que la transición de poder en 2014 no fue completamente democrática. En particular, desde el 2014 la libertad de los medios de comunicación se ha deteriorado significativamente. Después del Euromaidán no se ha mantenido ningún canal de televisión nacional o ningún periódico nacional que sea opositor al nuevo gobierno ucraniano. Las transmisiones de casi todos los canales de televisión rusos fueron prohibidas en Ucrania, igual que algunas páginas web y redes de comunicación (Katchanovski, 2016).

Según estudios realizados por la Oficina de la ONU de derechos humanos, con el inicio de la nueva campaña electoral para las elecciones presidenciales, que tuvieron lugar entre marzo y abril del 2019, se observó un incremento de los actos violentos contra periodistas, activistas de la sociedad civil y políticos (Civic space and fundamental freedoms ahead of the presidential, parliamentary and local elections in Ukraine in 2019-2020, 2019). Sin embargo, dichas elecciones fueron justas y sin violaciones significativas de la ley. El 21 de abril, se llevó a cabo la segunda ronda de elecciones presidenciales, donde el cómico Volodymyr Zelensky ganó con $73,22 \%$ de los votos a Petro 
Poroshenko, que recibió solamente $24,45 \%$ de los votos (" $\mathrm{La}$ Comisión Electoral Central anunció el resultado oficial de las elecciones presidenciales," 2019). Es un caso sin precedentes en la historia de Ucrania que un político no profesional gane las elecciones presidenciales. Este acto puede verse como una profunda desconfianza de los ucranianos hacia los políticos y el temor a una nueva autocracia en el país. No obstante, es demasiado pronto para especular sobre cómo la victoria de Zelensky afectará al futuro de Ucrania y sus relaciones con Rusia.

\section{$\underline{\text { Referencias }}$}

30 días de la guerra. ¿Cuál fue el motivo de la introducción de la ley marcial en Ucrania? (2018, November 27). Ukraiinska Pravda. Retrieved from https://www.pravda.com.ua/articles/2018/11/27/7199417/

Berkut empezó el ataque en Hrushevsky. (2014, January 22).

Ukraiinska Pravda. Retrieved from https://www.pravda.com.ua/news/2014/01/22/7010559/

Civic space and fundamental freedoms ahead of the presidential, parliamentary and local elections in Ukraine in 2019-2020. (2019). Retrieved from https://www.ohchr.org/Documents/Countries/UA/CivicSpace FundamentalFreedoms2019-2020.pdf

Durante los disturbios en Odessa 42 personas murieron. El jefe de policia fue despedido. (2014, May 2). Ukraiinska Pravda. Retrieved from https://www.pravda.com.ua/news/2014/05/3/7024232/

El barco de desembarco estadounidense USS FortMcHenry entró en el Mar Negro. (2019, January 7). Ukraiinska Pravda. Retrieved from https://www.pravda.com.ua/news/2019/01/7/7203146/

El consejo de Crimea ha nombrado un referéndum para unirse a la Federación Rusa para el 16 de marzo. (2014, March 6). Ukraïnska Pravda. Retrieved from 
https://www.pravda.com.ua/news/2014/03/6/7017808/

El Parlamento de Crimea nombró a un referéndum y dispersa el gobierno. (2014, February 27). Ukraiinska Pravda. Retrieved from https://www.pravda.com.ua/news/2014/02/27/7016540/

Gaidai, A. J., \& Liubaretc, A. V. (2016). LENINFALL:

ELIMINATION OF THE PAST AS A WAY OF

CONSTRUCTING THE FUTURE (ON THE MATERIALS

OF DNEPROPETROVSK, ZAPOROZHYE AND

KHARKOV). Vestnik Permskogo Universiteta. Istoriya, 2(33), 28-41.

https://doi.org/10.17072/2219-3111-2016-2-28-41

Katchanovski, I. (2016). The "Euromaidan," Democracy, and Political

Values in Ukraine. SSRN Electronic Journal.

https://doi.org/10.2139/ssrn.2835495

Kudryashev, I. (2015). El Conflicto de Ucraina en 2014: Causas y

consecuencias de la crisis. Anuario Del Conflicto Social, (4).

Retrieved from

http://revistes.ub.edu/index.php/ACS/article/view/12289/1504 3

Kuzio, T. (2016). Soviet and Russian anti-(Ukrainian) nationalism andre-Stalinization. Communist and Post-Communist Studies, 49(1), 87-99. https://doi.org/10.1016/j.postcomstud.2015.12.005

Kyrydon, A. M. (2015). EUROMAIDAN / REVOLUTION OF DIGNITY: THE CAUSES, NATURE, BASIC STAGES.

Istorychna Pamyat, 33, 17-32. Retrieved from

http://dspace.pnpu.edu.ua/bitstream/123456789/4395/1/Kirid on.pdf

La Comisión Electoral Central anunció el resultado oficial de las elecciones presidenciales. (2019, April 30). Ukraiinska Pravda.

Retrieved from

https://www.pravda.com.ua/news/2019/04/30/7213926/

La guerra en el estrecho de Kerch: barcos, aviones y captura de barcos 
ucranianos. (2018, November 25). Ukraïnska Pravda. Retrieved from

https://www.pravda.com.ua/articles/2018/11/25/7199243/

La policía puede volver a utilizar la fuerza contra manifestantes -

Ministerio del Interior. (2013, December 11). Ukraïnska Pravda.

Retrieved from

https://www.pravda.com.ua/news/2013/12/11/7006338/

Maidan. El nacimiento. (n.d.). Retrieved February 4, 2019, from https://www.pravda.com.ua/cdn/cd1/21nov/

Mityukov, N. V. (2016). The Periodization of Fighting on Donbass in 2014-2015 years. Voennyi Sbornik, 11(1), 43-48.

https://doi.org/10.13187/vs.2016.11.43

Nagornyak, T. L. (2015). The conflict between State and Society in Ukraine. Gileya, 88, 352-362. Retrieved from http://www.irbisnbuv.gov.ua/cgi-

bin/irbis_nbuv/cgiirbis_64.exe?C21COM=2\&I21DBN=UJRN\& P21DBN=UJRN\&IMAGE_FILE_DOWNLOAD=1\&Image_fil e_name=PDF/gileya_2014_88_87.pdf

Paniotto, V. I. (2013). Ukraina. Euromaidan. Vestnik Obshestvennogo Mneniya, 3-4(116), 17-23. Retrieved from https://cyberleninka.ru/article/n/ukraina-evromaydan

Putin firmó un decreto sobre la emisión de pasaportes rusos a los residentes de regiones de Donetsk y Luhansk. (2019, April 24). Ukraiinska Pravda. Retrieved from https://www.pravda.com.ua/news/2019/04/24/7213454/

Putin, V. (2014a). Address by President of the Russian Federation. Retrieved May 7, 2019, from President of Russia website: http://en.kremlin.ru/events/president/news/20603

Putin, V. (2014b). Línea directa con Vladimir Putin. Retrieved April 21, 2019, from President of Russia website: http://kremlin.ru/events/president/news/20796 
Radkovets Y. I. (2014). Síntomas de la tecnología de guerra híbrida en las acciones agresivas de Rusia contra Ucrania. Nauka i Oborona, (3), 36-42. Retrieved from http:/ /www.irbis-nbuv.gov.ua/cgibin/irbis_nbuv/cgiirbis_64.exe?I21DBN=LINK\&P21DBN=UJ $\mathrm{RN} \& Z 21 \mathrm{ID}=\& S 21 \mathrm{REF}=10 \& \mathrm{~S} 21 \mathrm{CNR}=20 \& \mathrm{~S} 21 \mathrm{STN}=1 \& \mathrm{~S} 21 \mathrm{FM}$ $\mathrm{T}=\mathrm{ASP} \_$meta\&C21COM=S\&2_S21P03 $=$FILA $=\& 2 \_S 21 \mathrm{STR}=$ na uio_2014_3_8

Report on the buman rights situation in Ukraine 16 November 2018 to 15

February 2019. (2019). Retrieved from

https://www.ohchr.org/Documents/Countries/UA/ReportUkra ine16Nov2018-15Feb2019.pdf

Report on the human rights situation in Ukraine from 1 December 2014 to 15

February 2015. (2015). Retrieved from

http://www.un.org.ua/images/stories/9thOHCHRreportUkraine _1.pdf

Report on the human rights situation in Ukraine from 16 August to 15

November 2015. (2015). Retrieved from

http://www.un.org.ua/images/stories/12th_OHCHR_report_on _Ukraine_EN.pdf

Report on the human rights situation in Ukraine from 16 August to 15 November 2016. (2016). Retrieved from

http://www.un.org.ua/images/ENG_16th_HRMMU_Report.pdf Report on the human rights situation in Ukraine from 16 August to 15

November 2017. (2017). Retrieved from

https://www.ohchr.org/Documents/Countries/UA/UAReport2 0th_EN.pdf

Report on the human rights situation in Ukraine from 16 August to 15

November 2018. (2018). Retrieved from

https://www.ohchr.org/Documents/Countries/UA/24thReport

UkraineAugust_November2018_EN.pdf

Sasse, G., \& Lackner, A. (2018). War and identity: the case of the 
Donbas in Ukraine War and identity: the case of the Donbas in Ukraine. Post-Soviet AffAirs, 34(3), 139-157.

https://doi.org/10.1080/1060586X.2018.1452209

Shveda, Y., \& Ho Park, J. (2016). Ukraine's revolution of dignity: The dynamics of Euromaidan. Journal of Eurasian Studies, 7(1), 85-91. Retrieved from

http://www.kci.go.kr/kciportal/ci/sereArticleSearch/ciSereArtiV iew.kci?sereArticleSearchBean.artiId=ART002333076\&locale $=$ en $\& S I D=E 12 V C G w o j 41 v X 6 p W V q s$

Sociopolis. (2014). Evaluación de los habitantes de Ucrania de la situación sociopolítica en el país a partir de enero de 2014.

Retrieved May 1, 2019, from http://sociopolis.ua/uk/doslidzhenya/doslidzhenya/174-sociopolitical-january-2014/

Surkov sobre los pasaportes de la Federación Rusa en regiones de Donetsk y Luhansk: es un deber a los hablantes de ruso. (2019, April 24). Ukraiinska Pravda. Retrieved from https://www.pravda.com.ua/news/2019/04/24/7213456/

Tipaldou, S., \& Casula, P. (2018). ¿Justificaciones populistas de la guerra? La intervención rusa en el este de Ucrania. Revista CIDOB d'Afers Internacionals, (119), 135-160. https://doi.org/10.24241/rcai.2018.119.2.135

Titushky dispararon a dos manifestantes - testigos. (2014, February 19). Ukraiinska Pravda. Retrieved from https://www.pravda.com.ua/news/2014/02/19/7014579/

Tres años de guerra en Donbas: eventos clave de ATO. (2017, April 6). Slovo i Dilo. Retrieved from https://www.slovoidilo.ua/2017/04/06/infografika/bezpeka/try -roky-vijny-donbasi-klyuchovi-podiyi-ato

Un destructor estadounidense entró en el mar negro. (2019, February 20). Ukraiinska Pravda. Retrieved from 
https://www.pravda.com.ua/news/2019/02/20/7207183/

Una marina naval de la Flota del Norte de la Federación Rusa entró en el Mar Negro. (2019, January 9). Ukraiinska Pravda. Retrieved from https://www.pravda.com.ua/news/2019/01/9/7203294/ 